\title{
Multiple-choice learning of line-drawn facial features: I. Inhibitory effects of observer scoring
}

\author{
MELVIN H. MARX \\ University of Missouri, Columbia, Missouri 65211
}

\begin{abstract}
Students in four high school psychology classes learned the names attached to line-drawn facial features. After each of three multiple-choice acquisition trials, subjects were also given transfer tests on names for the full faces (all four features together). A final retention test on all acquisition materials (individual features) was then given. In acquisition, subjects worked in pairs, alternately performing (selecting the names) or observing (watching paired performer respond). Correct names were shown by slide after all performers had responded. Observers in two of the classes scored paired performers' responses as correct or incorrect. The results revealed no reliable main effects for the observer-scoring variable but a number of reliable interactions. A highly generalized inhibitory effect in both acquisition and transfer could be attributed to the observer-scoring variable, with males but not females affected.
\end{abstract}

This experiment was performed to assess the effects on learning and transfer of observers' scoring of their paired partners' responses in a multiple-choice learning task in which the partners alternately perform (select the answers) or observe (watch their partners perform).

\section{METHOD}

\section{Subjects}

A total of 44 females and 44 males were trained and tested in four high school psychology classes in the normal classroom setting $(18,22,22$, and 26 student participants).

\section{Experimental Design}

The four classes were all trained on the individual facial features ( $f$ ) and were tested for transfer on the full faces (F). Observers in two of the classes scored their partners' responses (f-Fs), and observers in the other two classes did not (f-Fns).

\section{Procedure}

Subjects were trained in pairs, exchanging performance and observation roles. Correct answers were provided after responses were made. Subjects were first shown 16 female facial features ( 4 eyes, 4 mouths, 4 noses, and 4 hairlines) and then 16 male facial features. Performers were instructed to guess which of the four names provided (Flo, Ann, Pat, and Fay for the female

Thanks are due Gary Blodick, psychology teacher at Naples Senior High School, Naples, Florida, for his cooperation in this experiment. His four psychology classes were used as subjects, as part of the course work, to familiarize the students with psychological experimentation. In class periods subsequent to the collection of the data, the experiment was described and the nature of experimental psychology generally discussed by the experimenter. I am also indebted to Kathleen Marx for assistance in the collection of the data and to Monica Moore and George Seymour for assistance in the statistical analysis. This research was supported in part by the author's Research Career Award from the National Institute of Mental Health and by a grant from the U.S. Army Research Institute for the Behavioral and Social Sciences. The opinions expressed herein are those of the author and are in no way endorsed by the U.S. Army. features; Ken, Roy, Sam, and Bob for the male features) went with each feature. After all of the performers had written their answers, the correct answer was shown by slide. Features were shown in a random order.

As they entered the classroom, students were arranged in like-sex pairs, seated together. The student on the left was the performer for the first 16 training items, and the student on the right was the observer. They exchanged roles for the second set of items.

The students were told (1) that we were interested in comparing how observers learned and remembered relative to performers, so that observers should note the outcome for their paired performers' answers; (2) that the first trial would be pure guesswork and, because of the similarity of features, there would be difficulty in remembering some names on the later trials; and (3) that the work, while a part of their course work, would not count on their school marks in any way.

Observers in Group f-Fs placed a check mark by correct answers and a cross by incorrect answers; observers in Group f-Fns simply noted the outcomes.

Following a brief rest interval, the transfer test was administered, with all subjects seated and working individually. The four female faces were shown first, followed by the four male faces. The subjects were instructed to write in the name they thought belonged to each face. No knowledge of results was provided.

Three training-test trials were given in the same manner. At the conclusion of the third transfer test trial, a final retention test on the features was given without knowledge of results.

\section{RESULTS AND DISCUSSION}

The analysis of variance used on these data was 3 by 2 by 2 by 2 by 4 by 2 , with trials, sex of subject, same or different sex of facial stimuli, scoring or no scoring in observation, and class as between-subjects variables and performance/observation $(\mathrm{P} / \mathrm{O})$ as a withinsubjects variable. A slightly simpler analysis (no trials variable) was run for the retention test.

The observer-scoring variable was not a reliable main effect but showed a number of interactions (all reliable 
at $\mathrm{p}=.05$ or better). It interacted in transfer with (1) trials $[F(2,360)=3.70, \quad \mathrm{MSe}=1.24]$, (2) sex of subject $[F(1,360)=6.68$, MSe $=1.24]$, and $(3)$ sex of subject and $\mathrm{P} / \mathrm{O}[\mathrm{F}(1,360)=4.51, \mathrm{MSe}=1.24]$. Moreover, in the retention test of acquisition, for features rather than faces, scoring was a reliable main effect $[\mathrm{F}(1,72)=6.87, \mathrm{MSe}=4.61]$ and also interacted with sex of subject $[F(1,72)=3.84, \mathrm{MSe}=4.61]$. These various relationships are described below.

Observer scoring had a surprisingly generalized inhibitory effect on learning. Over trials, for example, scorers showed a main gain of only .53 correct transfer responses $(1.65,1.88$, and 2.18 mean values for Trials 1 , 2 , and 3 , respectively), whereas nonscorers showed a mean gain of 1.10 correct responses (corresponding mean values of $1.58,2.01$, and 2.68). On the final test for features, scorers correctly identified a mean of 8.20 features, compared with a mean of 9.74 correctly identified by nonscorers.

Evidence that this inhibitory effect was a genuine one, rather than simply a matter of any differences between the two sets of $\mathrm{f}-\mathrm{F}$ classes, is provided by the interactions, in the transfer data, of the scoring variable with sex of subject and $\mathrm{P} / \mathrm{O}$. Female subjects were not affected by the scoring task, showing mean correct response totals for the three transfer trials of 2.20 with scoring and 2.05 without scoring. Male subjects, on the other hand, were clearly inferior under the scoring condition, with only 1.54 mean correct facial identifications, compared with a corresponding nonscoring mean of 2.13 .

A similar interaction occurred in retention. Under no-scoring conditions, there was little male-female difference (means of 9.94 and 9.50 for males and females, respectively), whereas males correctly identified markedly fewer features that they had observed and scored (means of 7.43 and 8.85).

The triple interaction in transfer of observer scoring with sex of subject and $\mathrm{P} / \mathrm{O}$, plotted in Figure 1 , offers further, more specific evidence on the source of the inhibitory influence associated with observer scoring. The accentuated deficiency of male subjects under the observer-scoring condition indicates clearly that the inhibitory effects generalized from observed features to

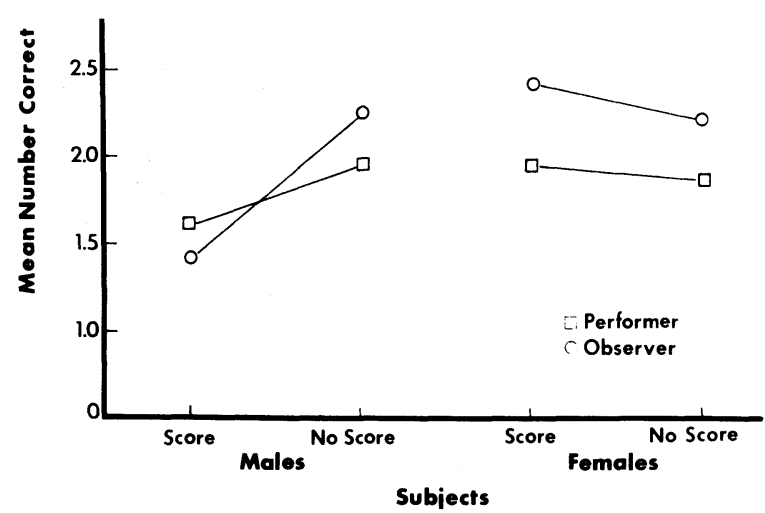

Figure 1. Mean number of correct faces in transfer for male and female subjects after performance or observation in training as a function of observer scoring or no scoring.

facial stimuli learned under performance, as well as being demonstrated on the observer stimulus materials themselves.

The importance of the sex difference demonstrated for the scoring variable is the suggestion that male high school students are more subject to distraction, at least in a verbal discrimination task of the kind used, than are female students, who are demonstrably superior observers and, presumably, superior rehearsers. How far this difference can be generalized across ages and tasks will, of course, need to be empirically determined, but the present data do offer an interesting starting point for further research with promise of practical as well as theoretical implications. Follow-up of any such leads may be regarded as especially important in light of the relative paucity of definitive data on sex differences and the current disagreement concerning them (e.g., Block, 1976; Maccoby \& Jacklin, 1974).

\section{REFERENCES}

BLock, J. H. Debatable conclusions about sex differences. Review of E. E. Maccoby and C. N. Jacklin, The psychology of sex differences. Contemporary Psychology, 1976, 21, 517-522.

MaCCOBY, E. E., \& J ACKLIN, C. N. The psychology of sex differences. Stanford, Calif: Stanford University Press, 1974.

(Received for publication September 21, 1979.) 